\title{
Holographically Probing Longitudinal Magnetic Fields with Electron Vortex Beams
}

Tyler R. Harvey ${ }^{1,2}$, Vincenzo Grillo ${ }^{3,4}$, Federico Venturi ${ }^{3,5}$, Jordan S. Pierce ${ }^{1}$, Fehmi S. Yasin ${ }^{1}$, Jordan J. Chess $^{1}$, Stefano Frabboni ${ }^{3,5}$, Ebrahim Karimi ${ }^{6,7}$ and Benjamin J. McMorran ${ }^{1}$

1. Department of Physics, University of Oregon, Eugene, OR, USA

2. IV. Physical Institute, University of Göttingen, Göttingen, Germany

3. CNR-Istituto Nanoscienze, Modena, Italy

4. CNR-IMEM, Parma, Italy

5. Dipartimento FIM, Universitá di Modena e Reggio Emilia, Modena, Italy

6. Department of Physics, University of Ottawa, Ottawa, ON, Canada

7. Department of Physics, Institute for Advanced Studies in Basic Sciences, Zanjan, Iran

Longitudinal magnetic fields play a key role in electron microscopes: the majority of electron lenses depend on a magnetic field oriented primarily along the electron propagation direction. However, electrons are unaffected by this component of the magnetic field, so one must tilt a specimen to obtain contrast from fields pointing out of the plane of the specimen. The combined contrast from the in-plane field, the tilted out-of-plane field and electrostatic potential variations in the material can be difficult to interpret without full three-dimensional reconstruction of the magnetic field and the specimen [1].

To avoid tilting, one can instead add transverse momentum to the electron beam. Adding linear momentum - tilting the illumination - is quite similar to tilting the sample. However, a electron with orbital angular momentum (a vortex beam) [2-4] is sensitive to a specimen's out-of-plane field at normal incidence. The collection of transverse momentum components that constitute the wavefunction of a vortex beam interact with a longitudinal field in such a way that the electron acquires an additional phase shift when compared to propagation over the same distance in vacuum. However, this phase shift of the vortex beam is only measurable if there is a reference wave with which to interfere.

Fortunately, the electron diffraction gratings that are a standard method to produce vortex beams in the first diffracted order also produce a zeroth-order beam without orbital angular momentum (OAM). When a diffraction grating is placed in the condenser aperture of a transmission electron microscope, and the condenser lenses are adjusted to produce focused beams in the specimen plane, the diffraction plane of the specimen then contains a hologram which is sensitive to relative phase shifts between the beams at the specimen. When the beams are raster-scanned across the specimen and a hologram is recorded at each probe position, this technique, called STEM holography [5-7], can produce phase-contrast images of the specimen that are similar to those produced by off-axis holography.

We employ STEM holography to retrieve the phase of a vortex beam at a single probe position to demonstrate the sensitivity to longitudinal magnetic fields [8]. We pass a vortex beam with $\pm 200 \hbar$ OAM around a $970 \mathrm{~nm}$-long FIB-deposited Co pillar and record a hologram for both plus and minus-OAM interacting with the pillar. We use a selected area aperture to block all beams with OAM of the opposite sign of the beam on the pillar. We then record holograms with the same beams on the bare substrate.

We use these holograms to calculate the difference in phase acquired by the $+200 \hbar$ and $-200 \hbar$ beams upon interacting with the pillar. This experimentally-determined phase agrees well with the value predicted from a Gaussian beam propagation model. We argue that this technique can be used to map the out-of-plane magnetic field of a specimen without tilting. Furthermore, as holograms recorded with both 
positive and negative OAM are sensitive to in-plane magnetic fields, we suggest that this technique provides the ability to simultaneously map all three components of the magnetic field produced by a specimen. The difference of positive and negative-OAM scans picks out the out-of-plane component, and the sum isolates the in-plane component, as well as electrostatic contrast. A second set of scans with a flipped specimen allows one to isolate electrostatic contrast from in-plane magnetic field contrast.

We have demonstrated that holographic reconstruction of the phase of electron vortex beams allows us to measure longitudinal magnetic fields. Full mapping of all three components of the magnetic field produced by specimens may become routine in the future using this technique [9].

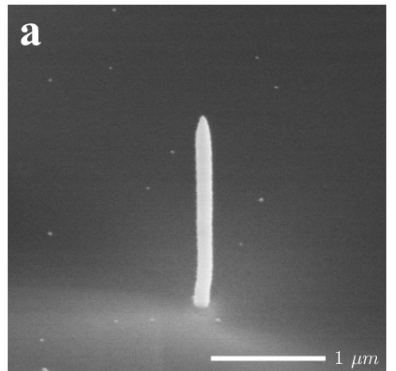

Figure 1. (a) Scanning electron micrograph of FIB-deposited Co pillar.

\section{References:}

[1] G. Lai et al., Journal of Applied Physics 75, 4593 (1998).

[2] M. Uchida and A. Tonomura, Nature 464, 737 (2010).

[3] J. Verbeeck, H. Tian, and P. Schattschneider, Nature 467, 301 (2010).

[4] B. J. McMorran, A. Agrawal, I. M. Anderson, A. A. Herzing, H. J. Lezec, J. J. McClelland, and J. Unguris, Science 331, 192 (2011).

[5] T. Leuthner et al., Phys. Stat. Sol. (A) 116, 113 (1989).

[6] J. M. Cowley, Ultramicroscopy 34, 293 (1990).

[7] F. S. Yasin et al., Microscopy and Microanalysis 22 (Suppl. 3), 506 (2016).

[8] V. Grillo, T. R. Harvey, F. Venturi, J. S. Pierce, R. Balboni, F. Bouchard, G. Carlo Gazzadi, S. Frabboni, A. H. Tavabi, Z.-A. Li, R. E. Dunin-Borkowski, R. W. Boyd, B. J. McMorran, and E. Karimi, Nature Communications 8, 689 (2017).

[9] Work supported by the U.S. DOE under Award DE-SC0010466. We gratefully acknowledge the use of CAMCOR facilities, which were purchased with a combination of federal and state funding.

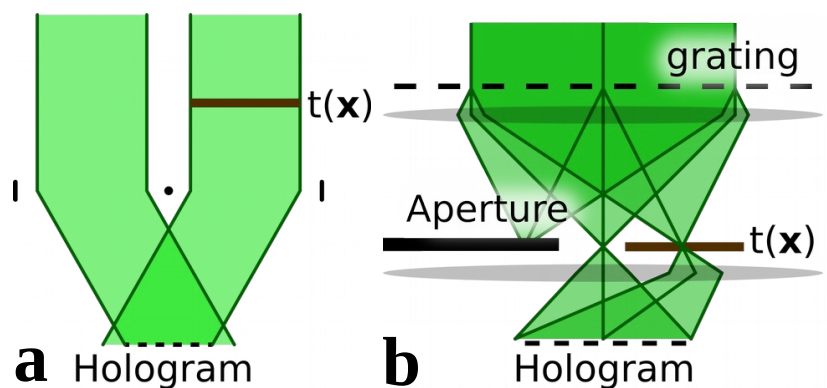

Figure 2. (a) Diagram of off-axis electron holography. A post-specimen biprism combines plane waves passed through vacuum and the specimen to form interference fringes in real space at the detector. (b) Diagram of STEM holography. A pre-specimen diffraction grating forms multiple probe beams at the specimen. These are re-combined with lenses. They form an interference pattern in the diffraction plane of the specimen. One interference pattern per probe position is recorded as the probes are scanned.
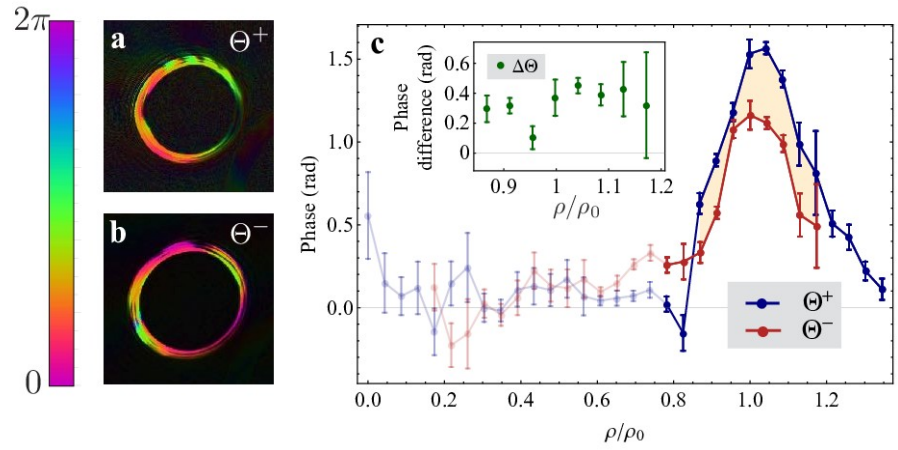

Figure 3. (a) Phase (color) and amplitude (brightness) reconstructed from hologram corresponding to $+200 \hbar$ beam interacting with magnetic pillar. (b) Same for $-200 \hbar$ beam. (c) Azimuthally-averaged phase vs. radius of phases shown in (a),(b). Inset: difference between phases.

This figure from Ref. [8] is licensed under CC-BY 4.0. http://creativecommons.org/licenses/by/4.0/ 